\title{
THE VIOLENT INTERSTELLAR MEDIUM IN MESSIER 31
}

\author{
Elias Brinks', Robert Braun², Stephen W. Unger ${ }^{1}$ \\ ${ }^{1}$ Royal Greenwich Observatory, Herstmonceux Castle, Hailsham BN27 1RP, England \\ ${ }^{2}$ National Radio Astronomy Observatory, Very Large Array, \\ Socorro, New Mexico 87801-0387, U.S.A.
}

\begin{abstract}
TAURUS observations in the line of $\mathrm{H} \alpha$ and VLA HI mapping of the HII complex No. 722 in M31, reveal what seems to be a spherical cavity $330 \mathrm{pc}$ in diameter blown out by a stellar association of over $20 \times 10^{6}$ year old. Evidence of induced star formation which was initiated less than $5 \times 10^{6}$ years ago is present in the form of bright HII emission and numerous O, B and Wolf-Rayet stars which are found within the shell surrounding the cavity. The energy necessary to create the HI shell is estimated to be about $5 \times 10^{51} \mathrm{erg}$.
\end{abstract}

\section{INTRODUCTION}

Vast amounts of energy are pumped into the interstellar medium (ISM) by an OB association, firstly in the form of photons and stellar winds by stars more massive than about $25 \mathrm{M}_{\odot}$, and later by supernova explosions of all stars heavier than about $8 \mathrm{M}_{\odot}$. One of the effects that this has is the creation of large cavities in the disks of spiral galaxies which are filled with hot gas and which are surrounded by expanding shells of ionised and neutral hydrogen (HI) gas. Clear evidence for this has been found by Heiles $(1979,1984)$ in our Galaxy and by Meaburn (1980) and Dopita et al. (1985) in the Large Magellanic Cloud. Recently Brinks and Bajaja (1986) have found similar features, HI holes, in the disk of the Andromeda galaxy in the HI survey of M31 with the Westerbork Synthesis Radio Telescope (WSRT). Very similar results have been derived for M33 on the basis of an HI survey with the WSRT by Deul (1988).

In recent years the standard two phase model of the ISM first proposed by Field, Goldsmith and Habing (1969) has been extended to describe a supernova dominated medium (Cox and Smith 1974; McKee and Ostriker 1977). These models consider three phases, hot, warm and cool gas, each roughly in pressure equilibrium. Later, several modifications have been suggested to account for the fact that the energy is not deposited uniformly throughout the disk but that the SN explode within the boundaries of their parent OB association (Heiles 1987; Norman and Ikeuchi 1989). As was mentioned by Heiles (this conference) there is considerable debate on the actual number of Type I and Type II supernovae, the number of OB associations, their distribution in the Galaxy, etc., which results in wildly varying predictions for the structure of the ISM and the filling factors of the various phases. Due to our unfavourable position within our own system it is unlikely that this situation will improve.

In order to complement the detailed but necessarily incomplete view which we have of our Galaxy we have undertaken a study at high linear resolution of M31 with the 
aim of providing better constraints for the input parameters of the different models. In addition, in M31 we can look at the structure of the ISM as a function of galactocentric distance. A further important justification to undertake this study is that we can inspect the interaction of an evolving $\mathrm{OB}$ association with its surroundings and test the various descriptions which have been put forward (see Tenorio-Tagle and Bodenheimer (1988) for a comprehensive review).

Below we will describe a set of observations obtained with a scanning Fabry-Perot interferometer in the line of $\mathrm{H} \alpha$, mapping the warm ionised component of the ISM. Our aim is to study the morphology of the $\mathrm{H} \alpha$ emission and its kinematics, notably the structure at the interface of the hot and ionised phase and the interaction between the ionised and cool components. Also, we will look for evidence of sites of secondary or induced star formation triggered by the violent events.

\section{OBSERVATIONS}

The observations that we report here were done with the TAURUS-I scanning Fabry-Perot interferometer at the Cassegrain focus of the 2.5-m Isaac Newton Telescope of the La Palma Observatory. They were conducted on 11/12 October 1987. For a description of the instrument and detector and information on the calibration and data reduction aspects the reader is referred to Bland et al. (1987). Eight fields in M31 were covered. A full account of the observations will be given elsewhere (Brinks et al., in preparation). Here we will highlight some results from one area. This field is located at a galactocentric distance of $9.7 \mathrm{kpc}$ in the northeastern part of the galaxy which is particularly rich in terms of HII regions and OB associations. It is centred at $34.2 \mathrm{~N}$ and 7.8E distance from the nucleus of M31 as measured in arcminutes along the North major and East minor axis. It covers the area of HII region P722, object No. 722 from the list of Pellet et al. (1978). The field of view of TAURUS on the INT is about 5 arcmin which at the assumed distance of M31 of $690 \mathrm{kpc}$ corresponds to $1000 \mathrm{pc}$. We mapped the line of $\mathrm{H} \alpha$ at a resolution of $\sim 8 \mathrm{~km} \mathrm{~s}^{-1}$. Our exposure time was $\sim 80 \mathrm{~min}$.

Figure 1 presents the sequence of channel maps which show in each frame the distribution of the $\mathrm{H} \alpha$ flux on the sky at a particular velocity. The central heliocentric velocity corresponding to each channel is indicated on top of each frame. The maps were smoothed to a resolution of 5 arcsec to increase the signal to noise ratio and to bring the resolution more in line with that of surveys of $\mathrm{HI}$ (see below). The equatorial (1950.0) coordinates of the maps were determined by measuring the positions of a set of foreground stars and are accurate to better than 1 arcsec. Channel to channel variations in the sky background due to terrestial emission lines such as $\mathrm{OH}$ at $\lambda=655.37$ and $656.90 \mathrm{~nm}$ have been removed by determining the background in areas free of line emission. A map representing the optical continuum emission has been constructed on the basis of those channels which do not contain any line emission. This map has been subtracted from the remaining channels.

\section{RESULTS AND DISCUSSION}

The $\mathrm{H} \alpha$ maps compare well with the photographs of Pellet et al. (1978) and shell structure corresponding to $\mathrm{P} 722$ can be readily identified. The entire area has a line width (FWHM) of $44 \mathrm{~km} \mathrm{~s}^{-1}$ and an integrated flux density of $2.5 \times 10^{-12} \mathrm{erg} \mathrm{cm}^{-2}$ $\mathrm{s}^{-1}$ (Walterbos and Braun, private communication). In $\mathrm{H} \alpha$ the shell is three quarters 

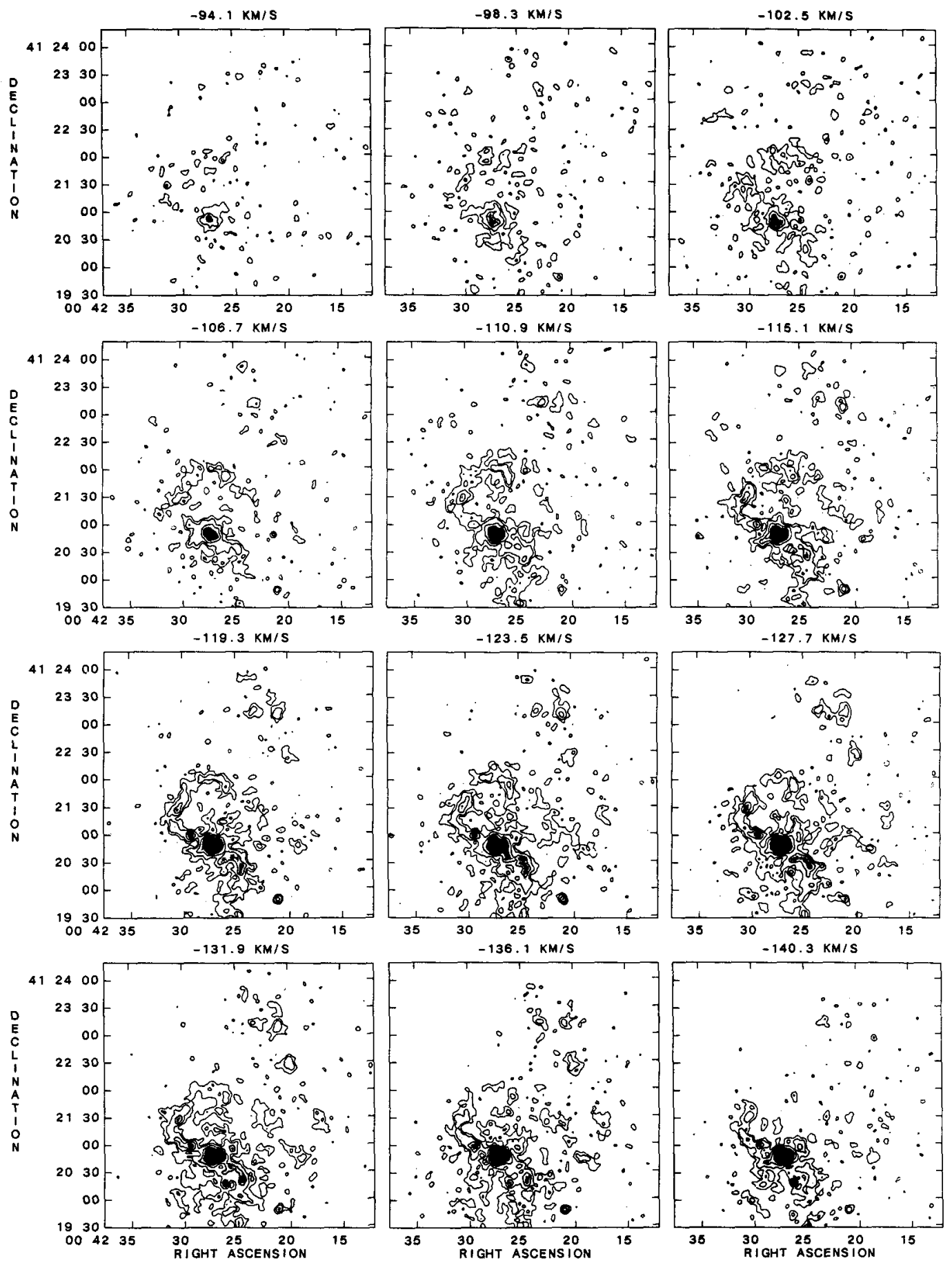

Figure 1: Sequence of $\mathrm{H} \alpha$ channel maps. The heliocentric velocity of each channel in $\mathrm{km} \mathrm{s}^{-1}$ is shown above each frame. Contour levels are at $-1,1,2,4,8$ and 16 in units of $2.14 \times 10^{-17} \mathrm{erg} \mathrm{cm}^{-2} \mathrm{~s}^{-1} \operatorname{arcsec}^{-2}$ per channel. The resolution is 5 arcsec, the channel separation $4.2 \mathrm{~km} \mathrm{~s}^{-1}$. 

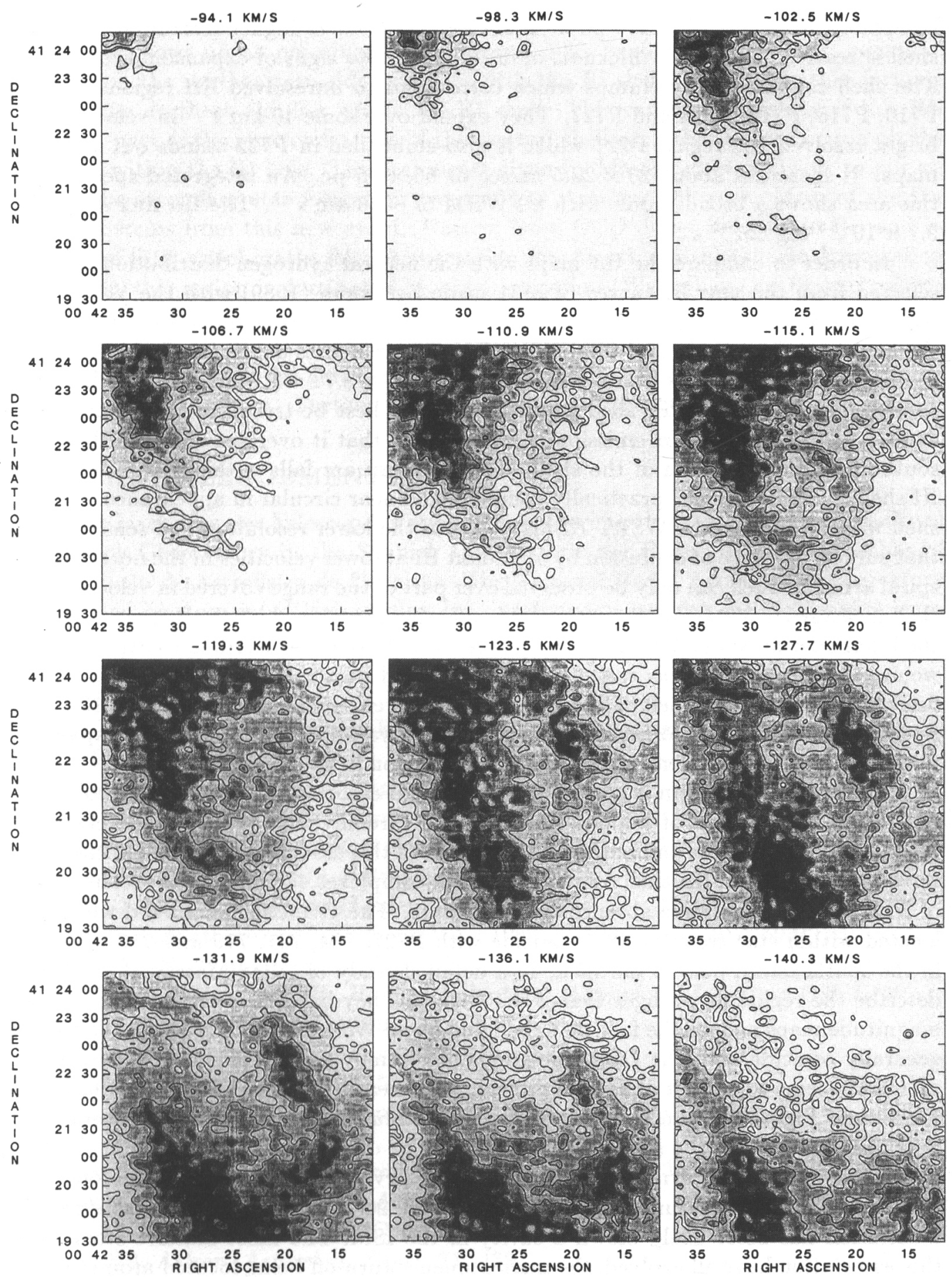

Figure 2: Sequence of HI channel maps at the same position and velocities as Figure 1. Contour levels are at $-1,1,1.4,2,2.8,4,5.6$, and 8 in units of $19.8 \mathrm{Kelvin}$. The rms noise level is $6.6 \mathrm{~K}$. The beam is elongated in the East-West direction and measures $11 \times 8.7$ arcsec. 
complete and measures $\sim 250 \mathrm{pc}$ in diameter. On a set of higher resolution maps the shell is resolved and has a thickness of order $20 \mathrm{pc}$. No signs of expansion are evident. The shell contains many clumps which correspond to unresolved HII regions such as P710, P716, P719, P724 and P727. They extend over some $40 \mathrm{~km} \mathrm{~s}^{-1}$ in velocity. The bright resolved HII region P721 which is also embedded in P722 stands out from the maps. It measures about $20 \times 22.5$ arcsec or $65 \times 75 \mathrm{pc}$. An integrated spectrum of this area shows a broad profile with a FWHM of $\sim 36 \mathrm{~km} \mathrm{~s}^{-1}$. The $\mathrm{H} \alpha$ flux density is $9.7 \times 10^{-13} \mathrm{erg} \mathrm{cm}^{-2} \mathrm{~s}^{-1}$.

In order to compare the $\mathrm{H} \alpha$ maps with the neutral hydrogen distribution we have selected from the new HI survey of M31 made by Braun (1989) with the Very Large Array (VLA) the same area. Figure 2 shows a sequence of HI channel maps on exactly the same coordinate grid and sampled at the same velocities as the $\mathrm{H} \alpha$ data cube. The resolution of the $\mathrm{HI}$ maps is $\Delta \alpha \times \Delta \delta \times \Delta V=11.0^{\prime \prime} \times 8.7^{\prime \prime} \times 5.1 \mathrm{~km} \mathrm{~s}^{-1}$. The $\mathrm{H} \alpha$ shell partly coincides with an HI shell whose outline can best be traced in the channel map at $-115.1 \mathrm{~km} \mathrm{~s}^{-1}$. The $\mathrm{H} \alpha$ emission is located such that it overlaps with the $\mathrm{HI}$ in the southern and eastern rim of the shell. The northern arc falls within the cavity. The $\mathrm{HI}$ shell is slightly larger, practically complete and near circular in appearance. The HI shell was not seen in the WSRT HI maps due to the lower resolution and sensitivity of that survey. Because of confusion by abundant $\mathrm{HI}$ at lower velocities in the northeastern spiral arm, the shell can only be observed over part of the range covered in velocity. The shell measures $\sim 330 \mathrm{pc}$ in diameter and is marginally resolved. As in $\mathrm{H} \alpha$, no change of shell diameter as a function of velocity is seen which could be ascribed to expansion. If we use the same method as employed by Brinks and Bajaja (1986) we derive an upper limit for the age of the shell of $20 \times 10^{6}$ year and an energy requirement of $5 \times 10^{51} \mathrm{erg}$. Because the shell is stationary we have assumed a value of $8 \mathrm{~km} \mathrm{~s}^{-1}$ for its expansion velocity which is equivalent to the velocity dispersion in the ISM.

The northeastern arm is particularly rich in $\mathrm{OB}$ associations and our field coincides with OB48 from the list of van den Bergh (1964). Efremov et al. (1987) have reclassified van den Bergh's objects and redrawn the borders of this association. Their photographs show that the $\mathrm{HI}$ and $\mathrm{H} \alpha$ shell coincide with star complexes 48-3 and 48-4. At the centre of P722 there is a relative absence of young stars. The OB associations a-d which are located within star complex 48-4 coincide with P727, 724, 721, 719 and 716 which lie in the southeastern part of the shell. In a detailed study of OB48 Massey et al. (1986) describe the region as younger than $5 \times 10^{6}$ year. They base their estimate on colourmagnitude maps and on the fact that they find seven Wolf-Rayet stars. These WR stars generally coincide with the $\mathrm{OB}$ associations of Efremov et al. According to Massey et al. OB48 seems to consist of several groups, each measuring about $200 \mathrm{pc}$ in diameter. From their Figure 3 it can be easily seen that the blue objects tend to be concentrated near the perimeter of the shell.

In an attempt to synthesize the various observations we arrive at the following picture. The oldest feature is the HI shell. Some $20 \times 10^{6}$ year ago an evolving OB association has most likely blown a cavity in the ISM. The most massive stars from this association have all evolved, the main sequence turn-off being located around $\mathrm{B} 0$ or at slightly later spectral types. Therefore no remnant association is seen by Massey $e t$ al. whose CCD photometry cuts off at stars older than B0. The HI shell, after having swept up a considerable amount of mass has stalled. Within the shell densities have 
reached high enough values to allow secondary star formation to commence. This star formation shows up as prominent HII regions. This explains why the $\mathrm{H} \alpha$ emission, notably on the southeastern side coincides with the HI shell. The $\mathrm{H} \alpha$ feature forming part of the northern rim lies within the HI shell. This can be understood if this HII region is part of the near or far side of the neutral shell such that its projection on the sky falls within the HI hole. The arguments given by Massey et al. allow us to put an age to the secondary star formation event of less than $5 \times 10^{6}$ year. Most of the $\mathrm{H} \alpha$ emission stems from this new event. Narrow band CCD direct imaging in $\mathrm{H} \alpha$ and in the line of [SII] corroborates this interpretation showing that although shock ionisation is present, this region is dominated by photoionisation (Walterbos and Braun, private communication).

These first results illustrate that it is feasible with current instruments to study the violent ISM in nearby galaxies at linear resolutions which could hitherto only be achieved in our Galaxy. Further analysis of this and the other fields is currently in progress.

\section{ACKNOWLEDGEMENTS}

The Isaac Newton Telescope is operated on the island of La Palma by the Royal Greenwich Observatory in the Spanish Observatorio del Roque de los Muchachos of the Instituto de Astrofisica de Canarias. The National Radio Astronomy Observatory is operated by Associated Universities, Inc., under contract with the National Science Foundation.

\section{REFERENCES}

Bland, J., Taylor, K., Atherton, P.D.: 1987, Monthly Notices Roy. Astron. Soc. 228, 595

Braun, R.: 1989, Astrophys. J. Supp. (in preparation)

Brinks, E., Bajaja, E.: 1986, Astron. Astrophys. 169, 14

Brinks, E., Burton, W.B.: 1984, Astron. Astrophys. 141, 195

Cox, D.P, Smith, B.W.: 1974, Astrophys. J. Lett. 189, L105

Deul, E.: 1988, Ph. D. Thesis (Leiden Observatory)

Dopita, M.A, Mathewson, D.S., Ford, V.L.: 1985, Astrophys. J. 297, 599

Efremov, Yu.N., Ivanov, G.R., Nikolov, N.S.: 1987, Astrophys. Space Sci. 135, 119

Field, G.B., Goldsmith, D.W., Habing, H.J.: 1969, Astrophys. J. Letters 155, L149

Heiles, C.: 1979, Astrophys. J. 229, 533

Heiles, C.: 1984, Astrophys. J. Suppl. 55, 585

Heiles, C.: 1987, Astrophys. J. 315, 555

Massey, P., Armandroff, T.E., Conti, P.S.: 1986, Astron. J. 92, 1303

McKee, C.F., Ostriker, J.P.: 1977, Astrophys. J. 218, 148

Meaburn, J.: 1980, Monthly Notices Roy. Astron. Soc. 192, 365

Norman, C.A., Ikeuchi, S.: 1989, Astrophys. J. (submitted)

Pellet, A., Astier, N., Viale, A., Courtès, G., Maucherat, A., Monnet, G., Simien, F.: 1978, Astron. Astrophys. Suppl. 31, 439

Tenorio-Tagle, G., Bodenheimer, P.: 1988, Ann. Rev. Astron. Astrophys. 26, 145

van den Bergh, S.: 1964, Astrophys. J. Suppl. 9, 65 